\title{
Tumor Identifier Character Result in Standard Format
}

National Cancer Institute

\section{Source}

National Cancer Institute. Tumor Identifier Character Result in Standard Format. NCI

Thesaurus. Code C117445.

The standard character or string for representation and reporting of tumor identification data. 